\title{
On the factorization of trajectory lifting maps
}

\author{
Paulo Tabuada \\ Dept. of Electrical Engineering \\ University of Notre Dame \\ Notre Dame, IN 46556 \\ E-mail: ptabuada@nd.edu
}

\begin{abstract}
Trajectory preserving and lifting maps have been implicitly used in many recursive or hierarchical control design techniques. Well known systems theoretic concepts such as differential flatness or more recent ones such as bisimulations can be also understood through the trajectory lifting maps they define. In this paper we initiate a study of trajectory preserving and lifting maps between affine control systems. Our main result shows that any trajectory lifting map between two single-input control affine systems can be locally factored as the composition of two special trajectory lifting maps: a projection onto a quotient system followed by a differentially flat output with respect to another control system.
\end{abstract}

\section{INTRODUCTION}

This paper initiates the study of a special class of maps between control systems having the property of preserving and lifting (or reflecting) trajectories. The importance of this class of maps can be recognized by realizing that several hierarchical or recursive control design techniques are implicitly based on the existence of such maps. The most popular example is probably backstepping [SJK97] where the existence of a stabilizing controller for a control system of the form:

$$
\dot{y}=f(y)+g(y) v
$$

with $y \in \mathbb{R}^{n}$ being the state and $v \in \mathbb{R}$ being the input can be extended to a stabilizing controller for the larger system:

$$
\begin{aligned}
& \dot{y}=f(y)+g(y) v \\
& \dot{v}=f^{\prime}(y, v)+g^{\prime}(y, v) u
\end{aligned}
$$

where $(y, v) \in \mathbb{R}^{n+1}$ is now the state, $u \in \mathbb{R}$ the input and $g^{\prime}$ is assumed to be non-zero in the region of interest. What is interesting in this design technique, from the perspective of this paper, is that we can define the map $\phi(y, v)=y$ from the state space of (I.2) to the state space (I.1) with the following two remarkable properties:

1) For any state trajectory $\mathrm{x}(t)=(\mathrm{y}(t), \mathrm{v}(t))$ of (I.2), $\phi(\mathrm{x}(t))=\mathrm{y}(t)$ is a trajectory of (I.1);

2) For any trajectory $y(t)$ of (I.1) there exists a trajectory $\mathrm{x}(t)$ of (I.2) such that $\phi(\mathrm{x}(t))=\mathrm{y}(t)$.

Indeed, if $\mathrm{x}(t)=(\mathrm{y}(t), \mathrm{v}(t))$ is a trajectory of (I.2) then $\mathrm{y}(t)=\phi(\mathrm{y}(t), \mathrm{v}(t))$ is the trajectory of (I.1) corresponding

This research was partially supported by the National Science Foundation CAREER award 0446716 to input $\mathrm{v}(t)$. Conversely, if $\mathrm{y}(t)$ is a trajectory of (I.1) then $(\mathrm{y}(t), \mathrm{v}(t))$ is the trajectory of (I.2) corresponding to input:

$$
\frac{\dot{\mathrm{v}}(t)-f^{\prime}(\mathrm{y}(t), \mathrm{v}(t))}{g^{\prime}(\mathrm{y}(t), \mathrm{v}(t))}
$$

and satisfying $\phi(\mathrm{y}(t), \mathrm{v}(t))=\mathrm{y}(t)$.

A different scenario where trajectory preserving and lifting maps also appear is in the study of abstractions of control systems initiated by Pappas and co-workers [PLS00]. Here, one starts with a control system $\Sigma_{F}$ defined on some manifold $M$ and a map $\phi: M \rightarrow N$ to some lower dimensional manifold and one seeks to construct a control system $\Sigma_{G}$ with state space $N$ such that $\phi$ has property (1). The motivation behind the construction of $\Sigma_{G}$ is that the lower dimensionality of $\Sigma_{G}$ renders its analysis simpler and hopefully properties studied in $\Sigma_{G}$ will lift to $\Sigma_{F}$ under the right technical assumptions. An instance of this approach is described in [TP05a] where the problem of designing trajectories for $\Sigma_{F}$ joining point $a$ to point $b$ is converted into the problem of designing trajectories for $\Sigma_{G}$ joining point $\phi(a)$ to point $\phi(b)$ followed by a constructive procedure lifting designed trajectories from $\Sigma_{G}$ to $\Sigma_{F}$.

Differential flatness can also be understood under the light of trajectory preserving and lifting maps. Given a differentially flat system $\Sigma_{F}$ equipped with a flat output $\phi: \mathbb{R}^{m} \rightarrow \mathbb{R}^{n}$ we can always construct the trivial control system $\Sigma_{G}$ on $\mathbb{R}^{n}$ defined by $\dot{y}=v$ where $y \in \mathbb{R}^{n}$ is the state and $v \in \mathbb{R}^{n}$ the input. Since any curve in $\mathbb{R}^{n}$ is a trajectory of $\Sigma_{G}$ we immediately have that $\phi$ satisfies property (1). Furthermore, being $\phi$ a flat output we also know that for every trajectory $\mathrm{y}(t)$ there exists a trajectory $\mathrm{x}(t)$ of $\Sigma_{F}$ satisfying $\phi(\mathrm{x}(t))=\mathrm{y}(t)$ which shows that (2) is also satisfied. However, more is true in this case. Not only trajectories of $\Sigma_{G}$ can be lifted to trajectories of $\Sigma_{F}$ as this lifting operation is unique, that is, for every trajectory $\mathrm{y}(t)$ of $\Sigma_{G}$ there is one and only one trajectory of $\Sigma_{F}$ mapping to $\mathrm{y}(t)$ under $\phi$. On the other extreme we have bisimilar control systems. If $\Sigma_{F}$ is bisimilar to control system $\Sigma_{G}$ through a relation defined by the graph of a map $\phi: M \rightarrow N$, then by definition ${ }^{1}$ of bisimulation, (1) is satisfied and every trajectory of $\Sigma_{G}$ can be lifted not to one but to a family of trajectories. In more detail we have that

\footnotetext{
${ }^{1}$ See for example [vdS04], [TP04], [Pap03] for a discussion of bisimulation in a systems theoretic context.
} 
for every trajectory $\mathrm{y}(t)$ of $\Sigma_{G}$ and for every point $x \in M$ satisfying $\phi(x)=\mathrm{y}(0)$ there exists a lifting trajectory $\mathrm{x}_{x}(t)$ of $\Sigma_{F}$ satisfying $\phi\left(\mathrm{x}_{x}(t)\right)=\mathrm{y}(t)$ and $\mathrm{x}_{x}(0)=x$. The situations just described correspond to two extreme cases since in general a trajectory preserving and lifting map does not admit unique liftings neither admits lifting for every possible initial condition. However, as we prove in this paper, every trajectory preserving and lifting map between singleinput control affine systems can be locally factored as the composition of two trajectory preserving and lifting maps of the kinds just described.

A related line of inquiry is the study of maps satisfying property (2) but not necessarily property (1) as was done in [Gra05] for the extreme case where trajectories can be lifted for all possible initial conditions. We believe that the results presented in this paper also offer some insight into this "one-sided" aspect of the question of which kinematic reductions [BLL02] can be seen as particular examples.

The results presented in this paper rely on the so called geometric approach to nonlinear control [Jur97], [NvdS95] and are presented in the setting of category theory [Lan71]. Even though category theory only plays a moderate role in the proof of our results, it the provides a convenient conceptual setting to study many problems in systems and control theory. Such approach has already been proved useful in the study of quotients [TP05b], bisimulations for dynamical, control and hybrid systems [HTP03], mechanical control systems [Lew00] as well as other problems in systems and control theory [Elk98]. Due to space limitations we were forced to eliminate the proofs of the most elementary results. The interested reader can consult such proofs in [Tab05].

\section{NOTATIONAL PRELIMINARIES}

We follow standard terminology and notation in differential geometry [AMR88]. We will assume all objects to be smooth unless stated otherwise and by smooth we mean infinitely differentiable. We will denote by $T M$ the tangent bundle of a manifold $M$ and by $T_{x} M$ the tangent space of $M$ at $x \in M$ spanned by $\left\{\frac{\partial}{\partial x_{1}}, \ldots, \frac{\partial}{\partial x_{m}}\right\}$ where $\left(x_{1}, \ldots, x_{m}\right)$ are the coordinates of $x$. Similarly we denote by $T f$ the tangent map of a map $f: M \rightarrow N$ while $T_{x} f$ denotes the tangent map of $f$ evaluated at $x \in M$. Recall that $T_{x} f$ maps tangent vectors in $T_{x} M$ to tangent vectors $T_{x} f \cdot X=$ $Y \in T_{f(x)} N$. For each $x \in M, T_{x} f \in L\left(\mathbb{R}^{m}, \mathbb{R}^{n}\right)$ where $L\left(\mathbb{R}^{m}, \mathbb{R}^{n}\right)$ denotes the space of linear maps from $\mathbb{R}^{m}$ to $\mathbb{R}^{n}$ and $m=\operatorname{dim}(M), n=\operatorname{dim}(N)$. When the dimension of the kernel of $T_{x} f$ does not change with $x$ we say that $f$ has constant rank. By an affine distribution we will mean a function assigning to each $x \in M$ a an affine space of $T_{x} M$. Recall that a subset $S$ of a vector space is said to be an affine space when for any $s, s^{\prime} \in S$ we have $\lambda s+\lambda^{\prime} s^{\prime} \in S$ for any $\lambda+\lambda^{\prime}=1$ and $\lambda, \lambda^{\prime} \in \mathbb{R}$. Similarly, a function $f(x, y)$ is said to be affine in $y$ when $f\left(x, \lambda y+\lambda^{\prime} y^{\prime}\right)=\lambda f(x, y)+\lambda^{\prime} f\left(x, y^{\prime}\right)$ in which case it can be written as $f(x, y)=\alpha(x)+\beta(x) u$. The exterior derivative of a real valued map $f$ will be denoted by $\mathbf{d} f$ while the Lie derivative of $f$ along vector field $X$ will be denoted by $L_{X} f$. Iterated Lie derivatives are defined by the recursion $L_{X}^{0} f=f$ and $L_{X}^{i+1} f=L_{X}\left(L_{X}^{i} f\right)$.

\section{THE CATEGORY OF AFFINE CONTROL SYSTEMS}

Informally speaking, a category is a collection of objects and morphisms between the objects and relating the structure of the objects. If one is interested in understanding vector spaces, it is natural to consider vector spaces as objects and linear maps as morphisms since they preserve the vector space structure. This choice for objects and morphisms defines Vect, the category of vector spaces. Choosing manifolds for objects leads to the the natural choice of smooth maps for morphisms and defines Man, the category of smooth manifolds. In this section we introduce the category of affine control systems which we regard as the natural framework to study trajectory lifting morphisms. Besides providing an elegant language to describe the constructions to be presented, category theory also offers a conceptual methodology for the study of objects, affine control systems, in this case. Since our results are of local nature we define affine control systems directly on open subsets of Euclidean space.

Definition 3.1: A local affine control system $\Sigma=$ $\left(M, \mathbb{R}^{o}, F\right)$ is defined by the following elements:

1) The state space $M$, an open subset of $\mathbb{R}^{m}$;

2) The input space $\mathbb{R}^{o}$;

3) The system map $F: M \times \mathbb{R}^{o} \rightarrow T M$ defined by:

$$
F(x, u)=X(x)+\sum_{i=1}^{o} Z_{i}(x) u_{i}
$$

where $x \in M, u=\left(u_{1}, \ldots, u_{o}\right) \in \mathbb{R}^{o}, X$ is a vector field on $M$ and $Z_{1}, \ldots, Z_{o}$ are linearly independent vector fields on $M$.

A local affine control system is said to be single-input when $o=1$.

Since we are working locally there is no loss in generality in assuming that vector fields $X, Z_{1}, \ldots, Z_{o}$ are globally defined in $M$. Furthermore, as we are interested in local results we will not distinguish between a control system $\Sigma_{F}$ and its restriction to an open subset $M^{\prime} \subset M$. The linear independence assumption also results in no loss of generality when the distribution spanned by $Z_{1}, \ldots, Z_{o}$ has constant rank. In this case if, for example, vector field $Z_{o}$ is linearly dependent on the remaining vector fields $Z_{1}, \ldots, Z_{o-1}$ we have $Z_{o}(x)=\sum_{i=1}^{o-1} c_{i}(x) Z_{i}(x)$ and the feedback $u_{i}=-c_{i}(x)+u_{i}^{\prime}$ can be used to cancel $Z_{o}$. The resulting control system $F^{\prime}\left(x, u^{\prime}\right)=X(x)+\sum_{i=1}^{o-1} Z_{i}(x) u_{i}^{\prime}$ can now be identified with a control system with input space $\mathbb{R}^{o-1}$ where the linear independence assumption is valid.

Definition 3.2: Let $\Sigma_{F}=\left(M, \mathbb{R}^{o}, F\right)$ and $\Sigma_{G}=$ $\left(N, \mathbb{R}^{p}, G\right)$ be affine control systems. A map $f=\left(f_{1}, f_{2}\right)$ : $M \times \mathbb{R}^{o} \rightarrow N \times \mathbb{R}^{p}$ with $f_{1}: M \rightarrow N$ and $f_{2}: M \times \mathbb{R}^{o} \rightarrow N$ is a morphism from $\Sigma_{F}$ to $\Sigma_{G}$ if the following equality holds:

$$
T_{x} f_{1}(x) \cdot F(x, u)=G\left(f_{1}(x), f_{2}(x, u)\right)
$$


To illustrate the notion of morphism consider affine control system $\Sigma_{F}$ defined by:

$$
\begin{aligned}
& \dot{x}_{1}=x_{1}^{2}+x_{1} x_{2} \\
& \dot{x}_{2}=x_{1} x_{2}^{2}+x_{1} u \\
& \dot{x}_{3}=x_{1}^{3} x_{2} x_{3}^{2}+x_{1} x_{3} u
\end{aligned}
$$

and affine control system $\Sigma_{G}$ defined by $\dot{y}=v$. To show that:

$$
\begin{aligned}
f_{1}\left(x_{1}, x_{2}, x_{3}\right) & =x_{1} \\
f_{2}\left(x_{1}, x_{2}, x_{3}, u\right) & =x_{1}^{2}+x_{1} x_{2}
\end{aligned}
$$

defines a morphism from $\Sigma_{F}$ to $\Sigma_{G}$ we need to show that (III.1) holds. We first note that $T_{x} f_{1}(x) \cdot F(x, u)=$ $x_{1}^{2}+x_{1} x_{2}$. Since $G\left(f_{1}(x), f_{2}(x, u)\right)=f_{2}(x)=x_{1}^{2}+x_{1} x_{2}$ we conclude that equality (III.1) is satisfied and that $f_{1}$ and $f_{2}$ define a morphism from $\Sigma_{F}$ to $\Sigma_{G}$.

The notion of morphism generalizes the notion of feedback equivalence so many times used in systems and control theory. Recall that control systems $\Sigma_{F}$ and $\Sigma_{G}$, defined by $F(x, u)=X(x)+\sum_{i=1}^{o} Z_{i}(x) u_{i}$ and $G(y, v)=Y(y)+$ $\sum_{i=1}^{o} W_{i}(y) v_{i}$, respectively, are said to be feedback equivalent when there exists a diffeomorphism in the state space $g(x)=y$ and an invertible feedback $h(x, v)=u=h_{x}(v)$ such that the feedback transformed system:

$$
\begin{gathered}
F^{\prime}(y, v)=T_{g^{-1}(y)} g \cdot X \circ g^{-1}(y) \\
+\sum_{i=1}^{o} T_{g^{-1}(y)} g \cdot Z_{i} \circ g^{-1}(y) h\left(g^{-1}(y), v\right)
\end{gathered}
$$

is equal to $G(y, v)$. Note that by using $x=g^{-1}(y)$ and $v=h_{x}^{-1}(u)$ the equality between $F^{\prime}(y, v)$ and $G(y, v)$ can be written as:

$T_{x} g \cdot X(x)+\sum_{i=1}^{o} T_{x} g \cdot Z_{i}(x) u=Y \circ g(x)+\sum_{i=1}^{o} W \circ g(x) h_{x}^{-1}(u)$

which is no more than (III.1) with $f_{1}(x)=g(x)$ and $f_{2}(x, u)=h_{x}^{-1}(u)$.

Local affine control systems introduced in Definition 3.1 and morphisms between local affine control systems introduced in Definition 3.2 define the category of local affine control systems denoted by $\mathbf{A C o n}_{\mathbf{l}}$. It follows from the affine nature of the considered control systems that morphisms are also affine in the following sense:

Proposition 3.3: Let $\Sigma_{F} \stackrel{f}{\longrightarrow} \Sigma_{G}$ be a morphism in $\operatorname{ACon}_{1}$. Then, $f_{2}(x, u)=\alpha(x)+\beta(x) \cdot v$ where $\alpha: M \rightarrow$ $\mathbb{R}^{p}$ and for each $x \in M, \beta(x) \in L\left(\mathbb{R}^{o}, \mathbb{R}^{p}\right)$.

Properties of affine control systems are sometimes easily studied with the help of a naturally induced affine distribution.

Definition 3.4: With each local affine control system $\Sigma$ we associate an affine distribution $\mathcal{A}$ defined by:

$$
\mathcal{A}(x)=X(x)+\operatorname{span}_{\mathbb{R}}\left\{Z_{1}(x), \ldots, Z_{o}(x)\right\}
$$

One can show that studying local affine control systems is in many ways equivalent to studying affine distributions and their morphisms [Elk98]. The essence of this correspondence is the following result that we will use later in the paper.

Proposition 3.5: Let $\Sigma_{F} \stackrel{f}{\longrightarrow} \Sigma_{G}$ be a morphism in ACon $_{1}$, then:

$$
T_{x} f_{1}(x)\left(\mathcal{A}_{F}(x)\right) \subseteq \mathcal{A}_{G} \circ f_{1}(x)
$$

Conversely, for any map $f_{1}: M \rightarrow N$ satisfying (III.7) there exists a unique map $f_{2}: M \times \mathbb{R}^{o} \rightarrow \mathbb{R}^{p}$ such that $f=\left(f_{1}, f_{2}\right)$ is a morphism from $\Sigma_{F}$ to $\Sigma_{G}$.

This correspondence between morphisms in $\mathbf{A C o n}_{\mathbf{l}}$ and affine distribution preserving maps critically relies on the affine structure of the control systems. For non-affine control systems additional assumptions are necessary to conclude regularity of $f_{2}$ as discussed in [Gra03].

\section{TRAJECTORIES OF AFFINE CONTROL SYSTEMS}

\section{A. The path subcategory}

Even tough we have already introduced the objects of study, affine control systems, and presented some of its properties we have not yet defined the fundamental notion of trajectory. Once again we will follow a categorical approach based on Joyal's and co-workers work on bisimulation [JNW96]. There are two main reasons for following this approach. One, is that this approach has alredeady proved useful in studying notions of bisimulation for dynamical, control and hybrid systems [HTP03]. The other reason, is that by altering the notion of path objects, defined below, we can use similar techniques to study different properties lifted by morphisms.

Definition 4.1: An object $\Sigma_{T}$ of $\mathbf{A C o n}_{\mathbf{l}}$ is a path object if the following hold:

1) $M$ is a connected subset of $\mathbb{R}$ containing the origin;

2) The input space is $\mathbb{R}^{0}=\{0\}$;

3) The system map $T$ is given by $T(t)=(t, 1)$.

A path or trajectory in a local affine control system $\Sigma_{F}$ is a morphism $\Sigma_{T} \stackrel{p}{\longrightarrow} \Sigma_{F}$.

Morphism $p=\left(p_{1}, p_{2}\right): \Sigma_{T} \rightarrow \Sigma_{F}$ captures the usual notion of trajectory since equality (III.1) reduces to:

$$
\frac{d}{d t} p_{1}(t)=T_{t} p_{1}(t) \cdot 1=F\left(p_{1}(t), p_{2}(t)\right)
$$

where we have identified the function $p_{2}$ defined on $M \times\{0\}$ with a function $p_{2}$ defined on $M$. The above definition is no more than an elegant way of expressing trajectories through the use of morphisms. At this point it is important to show that morphisms of control systems have property (1) mentioned in the Introduction. This immediately follows from our definition since given a path $\Sigma_{T} \stackrel{p}{\longrightarrow} \Sigma_{F}$ in $\Sigma_{F}$ and a morphism $\Sigma_{F} \stackrel{f}{\longrightarrow} \Sigma_{G}$ from $\Sigma_{F}$ to $\Sigma_{G}$ it follows immediately that $f \circ p$ is a morphism from $\Sigma_{T}$ to $\Sigma_{G}$, therefore a path in $\Sigma_{G}$.

\section{B. Path lifting morphisms}

Although morphisms in $\mathbf{A C o n}_{\mathbf{l}}$ preserve trajectories by construction not every morphism reflects or lifts trajectories. 
Definition 4.2: Let $\Sigma_{F} \stackrel{f}{\longrightarrow} \Sigma_{G}$ be a morphism in ACon $_{1}$. Morphism is said to be path lifting if for any path object $\Sigma_{T}$ and any morphism $\Sigma_{T} \stackrel{p}{\longrightarrow} \Sigma_{G}$ there exists a morphism $\Sigma_{T} \stackrel{p^{\prime}}{\longrightarrow} \Sigma_{F}$ making the following diagram commutative:

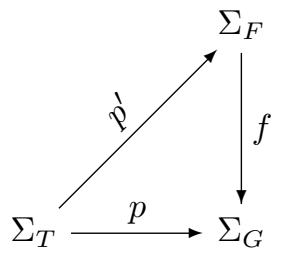

A path lifting morphism $f$ is said to be:

- Singular when $p^{\prime}$ is unique;

- Total when for every $x \in f_{1}^{-1}\left(p_{1}(0)\right)$ there exists a morphism $\Sigma_{T} \stackrel{p_{x}^{\prime}}{\longrightarrow} \Sigma_{F}$ making diagram (IV.1) commutative and satisfying $p_{x 1}^{\prime}(0)=x$.

It follows immediately from diagram (IV.1) that a necessary condition for $f$ to be a path lifting morphism is surjectivity of $f_{1}$. In addition to surjectivity other conditions must hold for a morphism to be path lifting. The study of such conditions requires the use of extensions of affine control systems introduced in the next section.

\section{EXTENSIONS}

The operation of extension allows to increase the state space dimension of a control system while retaining many of its properties. Extensions will play an important role in the factorization of path lifting morphisms.

Definition 5.1: Let $\Sigma=\left(M, \mathbb{R}^{o}, F\right)$ be a local affine control system. The extension of $\Sigma$, denoted by $\Sigma^{e}$, is defined by $\Sigma^{e}=\left(M^{e}, \mathbb{R}^{o}, F^{e}\right)$ where:

1) $M^{e}=M \times \mathbb{R}^{o}$;

2) $F^{e}((x, u), v)=X(x)+\sum_{i=1}^{o} Z_{i}(x) u_{i}+\sum_{i=1}^{o} v_{i} \frac{\partial}{\partial v_{i}}$. The extension of a control system models the addition of a pre-integrator to the original dynamics. If we start with a system of the form $\dot{x}=X(x)+Z_{1}(x) u_{1}+\ldots+Z_{o}(x) u_{o}$ its extension is described by:

$$
\begin{aligned}
\dot{x} & =X(x)+Z_{1}(x) u_{1}+\ldots+Z_{o}(x) u_{o} \\
\dot{u}_{1} & =v_{1} \\
& \vdots \\
\dot{u}_{o} & =v_{o}
\end{aligned}
$$

where $u_{1}, \ldots, u_{o}$ are now regarded as states and $v_{1}, \ldots, v_{o}$ are new inputs.

Note that the extension $\Sigma^{e}$ of a local affine control system comes equipped with a morphism $\Sigma^{e} \stackrel{\pi}{\longrightarrow} \Sigma$ defined by $\pi_{1}(x, u)=x$ and $\pi_{2}((x, u), v)=u$. Furthermore, morphism $\pi$ is a singular path lifting morphism since any trajectory $p(t)=\left(p_{1}(t), p_{2}(t)\right)$ in $\Sigma$ defines a unique trajectory $p^{e}(t)=$ $\left(\left(p_{1}(t), p_{2}(t)\right), \frac{d}{d t} p_{2}(t)\right)$ in $\Sigma^{e}$ satisfying $\pi \circ p^{e}=p$.

Proposition 5.2: Let $\Sigma_{F} \stackrel{f}{\longrightarrow} \Sigma_{G}$ be a morphism in $\mathbf{A C o n}_{\mathbf{l}}$ for $\Sigma_{F}=\left(M, \mathbb{R}^{o}, F\right)$ and $\Sigma_{G}=\left(N, \mathbb{R}^{p}, G\right)$, and assume that $T f_{1} \cdot Z_{i}=0$ for $i=1, \ldots, o$. Then, $f_{2}$ can be identified with a map $f_{2}: M \rightarrow \mathbb{R}^{p}$ and there exists a unique morphism $f^{e}$ making the following diagram commutative:

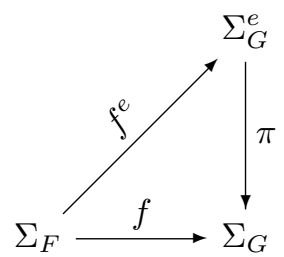

Furthermore, if $f$ is path lifting and $f_{1}$ has constant rank there exists a vector field $K$ defined on a neighborhood of every $x \in M$ satisfying $T f_{1} \cdot K=0$ and $T f_{1}^{e} \cdot K \neq 0$.

Proof: Since $f$ is a morphism we have $T_{x} f_{1}(x) \cdot F(x, u)=$ $G\left(f_{1}(x), f_{2}(x, u)\right)$ and assumption $T f_{1} \cdot Z_{i}=0$ implies that $T_{x} f_{1}(x) \cdot F(x, u)=T_{x} f_{1}(x) \cdot X(x)$. Therefore, for any $u, u^{\prime} \in \mathbb{R}^{o}$ it follows that $G\left(f_{1}(x), f_{2}(x, u)\right)=$ $G\left(f_{1}(x), f_{2}\left(x, u^{\prime}\right)\right)$. From injectivity of $G(y, v)$ in $v$ we conclude that $f_{2}(x, u)=f_{2}\left(x, u^{\prime}\right)$ so that we can identify $f_{2}$ with a function on $M$. Let now $f_{1}^{e}=\left(f_{1}, f_{2}\right): M \rightarrow$ $N \times \mathbb{R}^{p}$. If $V \in \mathcal{A}_{F}(x)$, then:

$$
\begin{aligned}
& T_{x} f_{1}^{e} \cdot V=\left(T_{x} f_{1} \cdot V, T_{x} f_{2} \cdot V\right) \\
= & \left(G\left(f_{1}(x), f_{2}(x)\right), T_{x} f_{2} \cdot V\right) \in \mathcal{A}_{G} \circ f_{1}(x) \times T_{f_{2}(x)} \mathbb{R}^{p} \\
= & \mathcal{A}_{G}^{e} \circ f_{1}^{e}(x)
\end{aligned}
$$

It now follows from Proposition 3.5 applied to $f_{1}^{e}$ the existence of a unique map $f_{2}^{e}$ making $\left(f_{1}^{e}, f_{2}^{e}\right)$ a morphism from $\Sigma_{F}$ to $\Sigma_{G}^{e}$. To conclude uniqueness of $f^{e}$ assume that $g$ is another morphism satisfying $\pi \circ g=f$. Since $\pi \circ g=g_{1}$ we conclude that $g_{1}=f=f_{1}^{e}$ and as $f_{e}^{2}$ is uniquely determined by $f_{1}^{e}=g_{1}$ it follows that $f^{e}=g$.

We now turn to the second part of the result and start by showing that if $f$ is path lifting then for every $x \in M$, $\left.f_{2}\right|_{L}$ is surjective where $L$ is the submanifold ${ }^{2} L=f_{1}^{-1} \circ$ $f_{1}(x)$ of $M$. For any trajectory $p$ in $\Sigma_{G}$ starting at $f_{1}(x)$, there exists a trajectory $p^{\prime}$ of $\Sigma_{F}$ satisfying $f \circ p^{\prime}=p$, by assumption. Differentiating $f_{1} \circ p_{1}^{\prime}=p_{1}$ at $t=0$ we get $T_{x} f_{1}(x) \cdot \dot{p}_{1}^{\prime}(0)=\dot{p}_{1}(0)$. Since $\dot{p}_{1}(0)$ can be any vector in $\mathcal{A}_{G} \circ f_{1}(x)$, there must exist a $x^{\prime} \in M$ such that $T_{x^{\prime}} f_{1}$. $F\left(x^{\prime}, u\right)=G\left(f_{1}\left(x^{\prime}\right), f_{2}\left(x^{\prime}\right)\right)=\dot{p}_{1}(0)$ and $f_{1}\left(x^{\prime}\right)=f_{1}(x)$, that is $x^{\prime} \in L$. We thus conclude that $\left.f_{2}\right|_{L}$ must be surjective in order for $\mathcal{A}_{G} \circ f_{1}(x)$ to be contained in the image of $G\left(f_{1}\left(x^{\prime}\right), f_{2}\left(x^{\prime}\right)\right)$ with $x^{\prime} \in L$ since $G(y, v)$ is injective on $v$. Having proved surjectivity of $\left.f_{2}\right|_{L}$ we now assume, for the sake of contradiction, that no vector field $K$ satisfies $T f_{1}$. $K=0$. But this implies that $L$ is a manifold of dimension 0 since the tangent space of $L$ is described by the vector fields $V$ satisfying $T f_{1} \cdot V=0$. We thus reach a contradiction since level set $L$ has at most a countable number of connected components which prevents $\left.f_{2}\right|_{L}$ from being surjective (on the codomain $\mathbb{R}$ ). Therefore we conclude the existence of vector fields $K$ satisfying $T f_{1} \cdot K=0$ and to finalize the proof we assume, again for the sake of contradiction, that every vector field satisfying $T f_{1} \cdot K=0$ also satisfies $T f_{2}$. $K=0$. However, this assumption implies that $f_{2}$ is constant

\footnotetext{
${ }^{2}$ Recall that since $f_{1}$ has constant rank $L=f_{1}^{-1} \circ f_{1}(x)$ is a submanifold of $M$.
} 
on every connected component of $L$ since the tangent space of $L$ consists of all vector fields $V$ satisfying $T f_{1} \cdot V=$ 0 . Furthermore, since $L$ has at most a countable number of connected components we contradict again surjectivity of $\left.f_{2}\right|_{L}$ thus finishing the proof.

\section{MAIN RESULT}

In this section we present and prove our main result. Its statement requires a variation on the notion of relative degree usually found in the geometric control theory literature [Isi96], [NvdS95]. The slightly different notion presented here will simplify the statement of the main results.

Definition 6.1: Let $\Sigma_{F} \stackrel{f}{\longrightarrow} \Sigma_{G}$ be a morphism in $\mathbf{A C o n}_{\mathbf{l}}$ where $\Sigma_{F}$ and $\Sigma_{G}$ are single-input systems. The relative degree of $\Sigma_{F}$ with respect to $f$ is the natural number $k$ satisfying:

1) $k=0$ if $T f_{1} \cdot Z \neq 0$ otherwise:

2) $k=1$ if $L_{Z} f_{2} \neq 0$;

3) $k=i+1$ if $L_{Z} L_{X}^{j} f_{2}=0$ for $j=0, \ldots, i-1$ and $L_{Z} L_{X}^{i} f_{2} \neq 0$.

Note that the relative degree is not necessarily well defined at every point in the state space. However, since our results are local in nature, we will assume that the state space has been reduced in order to contain only points where the relative degree is well defined.

Theorem 6.2: Let $\Sigma_{F} \stackrel{f}{\longrightarrow} \Sigma_{G}$ be a path lifting morphism in $\mathbf{A C o n}$ where $\Sigma_{F}$ and $\Sigma_{G}$ are single input systems. If $\Sigma_{F}$ has relative degree $k$ with respect to $f$ and $f_{1}$ has constant rank, then there exists a unique total path lifting morphism $\Sigma_{F} \stackrel{h}{\longrightarrow} \Sigma_{G}^{e^{k}}$ and a unique singular path lifting morphism $\Sigma_{G}^{e^{k}} \stackrel{g}{\longrightarrow} \Sigma_{G}$ making the following diagram commutative:

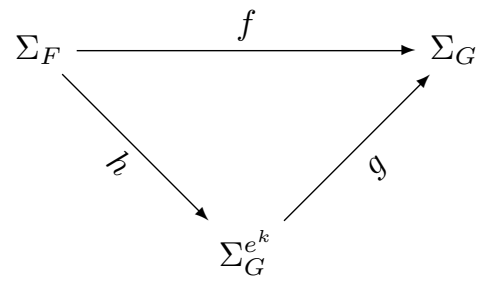

Furthermore, $g=\left(g_{1}, g_{2}\right)$ is given by the natural projections on the first factor $g_{1}: N \times \mathbb{R}^{k p} \rightarrow N$ and $g_{2}:\left(N \times \mathbb{R}^{p}\right) \times$ $\mathbb{R}^{k p} \rightarrow N \times \mathbb{R}^{p}$.

Proof: We start by considering the case where $T f_{1} \cdot Z \neq$ 0 , that is $k=0$. Let $F(x, u)=X(x)+Z(x) u$ and $G(y, v)=Y(y)+W(y) v$ and recall that by Proposition 3.3, $f_{2}(x, u)=\alpha(x)+\beta(x) u$. Evaluating $T_{x} f_{1} \cdot F(x, u)=$ $G\left(f_{1}(x), f_{2}(x, u)\right)$ at $u=0$ provides:

$$
T_{x} f_{1} \cdot X(x)=Y \circ f_{1}(x)+W \circ f_{1}(x) \alpha(x)
$$

Evaluating now $T_{x} f_{1} \cdot F(x, u)=G\left(f_{1}(x), f_{2}(x, u)\right)$ for an arbitrary $u \in \mathbb{R}$ and using (VI.1) we obtain:

$$
T_{x} f_{1} \cdot Z(x)=W \circ f_{1}(x) \beta(x)
$$

Since the left hand side is, by assumption, nonzero it follows that $\beta(x)$ must also be nonzero. We can therefore consider the feedback equivalent system $\Sigma_{F^{\prime}}$ defined by $F^{\prime}\left(x, u^{\prime}\right)=$
$F\left(x, \frac{u^{\prime}-\alpha(x)}{\beta(x)}\right)=X^{\prime}(x)+Z^{\prime}(x) u^{\prime}$. Note that $f$ is also a morphism from $\Sigma_{F^{\prime}}$ to $\Sigma_{G}$ and equality $T_{x} f_{1} \cdot F^{\prime}\left(x, u^{\prime}\right)=$ $G\left(f_{1}(x), f_{2}\left(x, u^{\prime}\right)\right)$ now reduces to:

$$
\begin{aligned}
& T_{x} f_{1} \cdot X^{\prime}(x)+T_{x} f_{1} \cdot Z^{\prime}(x) u^{\prime} \\
= & Y \circ f_{1}(x)+W \circ f_{1}(x) \alpha(x) \\
& +W \circ f_{1}(x) \beta(x) \frac{u^{\prime}-\alpha(x)}{\beta(x)} \\
= & Y \circ f_{1}(x)+W \circ f_{1}(x) u^{\prime}
\end{aligned}
$$

Let now $p(t)=\left(p_{1}(t), p_{2}(t)\right)$ be any trajectory in $\Sigma_{G}$ starting at any $y \in N$, that is, $p_{1}(0)=y$. Consider also the trajectory $p^{\prime}(t)$ in $\Sigma_{F^{\prime}}$ satisfying $p_{2}^{\prime}=p_{2}$ and starting at any $x \in M$ such that $f_{1}(x)=y$, that is, $p_{1}^{\prime}(0)=x$. Differentiating equality $f_{1} \circ p_{1}^{\prime}(t)=p_{1}(t)$ with respect to time and using (VI.2) we obtain:

$$
\begin{aligned}
\frac{d}{d t} f \circ p_{1}^{\prime}(t) & =T_{p_{1}^{\prime}(t)} f_{1} \cdot X^{\prime} \circ p_{1}^{\prime}(t)+Z^{\prime} \circ p_{1}^{\prime}(t) p_{2}^{\prime}(t) \\
& =Y \circ f_{1}\left(p_{1}^{\prime}(t)\right)+W \circ f_{1}\left(p_{1}^{\prime}(t)\right) p_{2}^{\prime}(t) \\
& =Y \circ f_{1}\left(p_{1}^{\prime}(t)\right)+W \circ f_{1}\left(p_{1}^{\prime}(t)\right) p_{2}(t)
\end{aligned}
$$

thus showing that $f \circ p_{1}^{\prime}(t)$ is the trajectory of $\Sigma_{G}$ corresponding to input $p_{2}(t)$. Since trajectories are necessarily unique it follows that we must have $f_{1} \circ p_{1}^{\prime}(t)=p_{1}(t)$ from which we conclude that for every trajectory $p(t)$ in $\Sigma_{G}$ starting at any $y \in N$ and for any $x \in M$ satisfying $f_{1}(x)=y$ there exists a trajectory $p^{\prime}(t)$ in $\Sigma_{F^{\prime}}$ starting at $x$ and satisfying $f_{1} \circ p^{\prime}=p$. Morphism $f$ is therefore a total path lifting morphism from $\Sigma_{F^{\prime}}$ to $\Sigma_{G}$ and therefore also a total path lifting morphism from $\Sigma_{F}$ to $\Sigma_{G}$ as $\Sigma_{F}$ is isomorphic to $\Sigma_{F^{\prime}}$.

We now consider the case where $T f_{1} \cdot Z=0$. By assumption $f_{1}$ has constant rank so that we can apply Proposition 5.2 to factor $\Sigma_{F} \stackrel{f}{\longrightarrow} \Sigma_{G}$ as $\Sigma_{F} \stackrel{f^{e}}{\longrightarrow} \Sigma_{G}^{e} \stackrel{\pi}{\longrightarrow} \Sigma_{G}$. Recall that $f_{1}^{e}=\left(f_{1}, f_{2}\right)$ and since by Proposition 5.2 there exists a vector field $K$ such that $T f_{1} \cdot K=0$ and $T f_{1}^{e} \cdot K \neq 0$ we conclude that $T f_{2} \cdot K \neq 0$. This shows that $\mathbf{d} f_{2}$ is linearly independent of $\mathbf{d} h_{1}, \ldots, \mathbf{d} h_{n}$ for any coordinate description $f_{1}=\left[\begin{array}{lll}h_{1} & \ldots & h_{n}\end{array}\right]^{T}$ of $f_{1}$. Therefore, $\operatorname{dim} \operatorname{ker}\left(T f_{1}^{e}\right)=\operatorname{dim} \operatorname{ker}\left(T f_{1}\right)-1$ and $f_{1}^{e}=\left(f_{1}, f_{2}\right)$ has constant rank since $f_{1}$ has constant rank. Note also that if the relative degree of $\Sigma_{F}$ with respect to $f$ is greater than one we have $L_{Z} f_{2}=0$ which combined with $T f_{1}$. $Z=0$ implies $T f_{1}^{e} \cdot Z=0$. We can therefore apply Proposition 5.2 again to factor $\Sigma_{F} \stackrel{f^{e}}{\longrightarrow} \Sigma_{G}^{e} \stackrel{\pi}{\longrightarrow} \Sigma_{G}$ as $\Sigma_{F} \stackrel{f^{e^{2}}}{\longrightarrow} \Sigma_{G}^{e^{2}} \stackrel{\pi^{2}}{\longrightarrow} \Sigma_{G}^{e} \stackrel{\pi}{\longrightarrow} \Sigma_{G}$. We now have $f_{1}^{e^{2}}=f^{e}=\left(f, T f_{2} \cdot F\right)$ since by Proposition $5.2 f^{e}$ is the unique morphism determined by $f$ and $f^{e}$ is a morphism as can be seen from:

$T f^{e} \cdot F=\left(T f_{1} \cdot F, T f_{2} \cdot F\right)=\left(G \circ f, T f_{2} \cdot F\right)=G^{e} \circ f^{e}$

Provided that the relative degree of $\Sigma_{F}$ is greater than 2 , it follows that $L_{Z} L_{X} f_{2}=0=L_{Z}\left(T f_{2} \cdot X\right)=L_{Z}\left(f_{2}^{e}\right)=$ $T f_{2}^{e} \cdot Z=0$ leading to $T f_{1}^{e^{2}} \cdot Z=0$. Also since there exists a vector field $K$ satisfying $T f_{1}^{e} \cdot K=0$ and $T f_{1}^{e^{2}} \cdot K \neq 0$ we conclude that $\operatorname{dim} \operatorname{ker}\left(T f_{1}^{e^{2}}\right)=\operatorname{dim} \operatorname{ker}\left(T f_{1}^{e}\right)-1=$ 
$\operatorname{dim} \operatorname{ker}\left(T f_{1}\right)-2$ and $f_{1}^{e^{2}}$ has constant rank. We can thus apply Proposition 5.2 repeatedly for a total of $k$ times after which $T f_{1}^{e^{k}} \cdot Z \neq 0$ since $f_{1}^{e^{k}}=\left(f_{1}^{e^{k-1}}, L_{X}^{k-1} f_{2}\right)$ and by definition of relative degree we have $L_{Z} L_{X}^{k-1} f_{2} \neq 0$. We thus have $f=f^{e^{k}} \circ \pi^{k} \circ \ldots \circ \pi^{2} \circ \pi$ with $\Sigma_{G}^{e^{i}} \stackrel{\pi^{i}}{\longrightarrow} \Sigma_{G}^{e^{i-1}}$. Morphism $g$ is now given by $g=\pi \circ \pi^{2} \circ \ldots \circ \pi^{k}$ and the proof is finished by defining $h=f^{e^{k}}$ and noting that $f^{e^{k}}$ is a total path lifting morphism (by the argument in the first part of the proof) since the relative degree of $\Sigma_{F}$ with respect to $f^{e^{k}}$ is zero.

Even tough a general path lifting morphism $\Sigma_{F} \stackrel{f}{\longrightarrow} \Sigma_{G}$ is neither total or singular Theorem 6.2 asserts that $f$ can be uniquely factored into a composition of singular and total path lifting morphisms. This decomposition allows one to regard $\Sigma_{F}$ as a control system that is differentially flat with respect to $\Sigma_{G}$ up to symmetries. As explained in [TP04], a total path lifting morphism $\Sigma_{F} \stackrel{h}{\longrightarrow} \Sigma_{G}^{e^{k}}$ that is also a surjective submersion necessarily corresponds to the projection from $\Sigma_{F}$ onto the quotient control system $\Sigma_{G}^{e^{k}}$ obtained from $\Sigma_{F}$ by factoring out the controlled invariant distribution defined by all the vector fields $K$ satisfying $T h_{1} \cdot K=0$. Once this controlled invariant distribution, describing symmetries of $\Sigma_{F}$, is factored out we obtain a singular path lifting morphism $\Sigma_{G}^{e^{k}} \stackrel{g}{\longrightarrow} \Sigma_{G}$ that can be regarded as a differentially flat output with respect to $\Sigma_{G}$ in the sense that any trajectory of $\Sigma_{G}$ lifts uniquely to a trajectory of $\Sigma_{G}^{e^{k}}$. The special cases of singular and total path lifting morphisms correspond to the cases where $h$ or $g$ are the identity morphisms, respectively, as we now summarize in the following corollary.

Corollary 6.3: Let $\Sigma_{F} \stackrel{f}{\longrightarrow} \Sigma_{G}$ be a path lifting morphism in $\mathbf{A C o n}$ where $\Sigma_{F}$ and $\Sigma_{G}$ are single input systems.

1) If $\Sigma_{F}$ has relative degree 0 with respect to $f$ then $f$ is a total path lifting morphism;

2) If $\Sigma_{F}$ has relative degree $\operatorname{dim}(M)-\operatorname{dim}(N)$ with respect to $f$ then $f$ is a singular path lifting morphism.

We now apply Theorem 6.2 to factor morphism $f$ defined by (III.5) and (III.6) as $f=g \circ h$. We first compute the relative degree $k$ of $\Sigma_{F}$, defined by (III.2), (III.3) and (III.4), with respect to $f$. Since $T f_{1} \cdot Z=0$ and $L_{Z} f_{2}=x_{1}^{2}$ we conclude that $k=1$ provided that $M$ is any open subset of $\mathbb{R}^{3}$ not containing the hyper-plane defined by $x_{1}=0$. Since $k=1$ Theorem 6.2 reduces to Proposition 5.2 and $h$ is the morphism $\Sigma_{F} \stackrel{f^{e}}{\longrightarrow} \Sigma_{G}^{e}$ while $g$ is the projection morphism $\Sigma_{G}^{e} \stackrel{\pi}{\longrightarrow} \Sigma_{G}$. From the proof of Proposition 5.2 (see [Tab05]), $f_{1}^{e}$ is given by $f_{1}^{e}=\left(f_{1}, f_{2}\right)$ and we can find $f_{2}^{e}$ through equality (III.1). Comparing:

$$
T f_{1}^{e} \cdot F=\left[\begin{array}{c}
x_{1}^{2}+x_{1} x_{2} \\
2 x_{1}^{3}+x_{1} x_{2}\left(3 x_{1}+x_{2}+x_{1} x_{2}\right)+x_{1}^{2} u
\end{array}\right]
$$

with $G^{e}\left(f_{1}^{e}(x), f_{2}^{e}(x, u)\right)$ we conclude that $f_{2}^{e}$ is given by:

$$
f_{2}^{e}\left(x_{1}, x_{2}, x_{3}, u\right)=2 x_{1}^{3}+x_{1} x_{2}\left(3 x_{1}+x_{2}+x_{1} x_{2}\right)+x_{1}^{2} u
$$

We can therefore regard $\Sigma_{F}$ as a differentially flat system with respect to the output $f_{1}$ modulo the symmetries defined by the controlled invariant distribution $\operatorname{ker}\left(T f_{1}^{e}\right)$.

\section{CONCLUSIONS}

The results described in this paper constitute the first step to understand and place in a broader setting the many existing hierarchical and recursive control design algorithms. Even though only the single-input case has been discussed we believe that a similar decomposition result should hold also for the multi-input case. In addition to a study of the multiinput case, ongoing research is focusing on the study of weaker forms of path lifting in order to extend hierarchical and recursive control design techniques to broader classes of systems.

\section{REFERENCES}

[AMR88] R. Abraham, J. Marsden, and T. Ratiu. Manifolds, Tensor Analysis and Applications. Applied Mathematical Sciences. Springer-Verlag, 1988.

[BLL02] F. Bullo, A. D. Lewis, and K. M. Lynch. Controllable kinematic reductions for mechanical systems: concepts, computational tools, and examples. In Proceedings of 15th International Symposium on Mathematical Theory of Networks and Systems, Notre Dame, IN, August 2002.

[Elk98] V. I. Elkin. Affine control systems: Their equivalence, classification, quotient systems, and subsystems. Journal of Mathematical Sciences, 88(5):675-721, 1998.

[Gra03] K. A. Grasse. Admissibility of trajectories for control systems related by smooth mappings. Mathematics of Control, Signals and Systems, 16(2):120-140, 2003.

[Gra05] K. A. Grasse. Lifting of trajectories of control systems related by smooth mappings. Systems and Control Letters, 2005. In press.

[HTP03] Esfandiar Haghverdi, Paulo Tabuada, and George Pappas. Bisimulation relations for dynamical and control systems. In Rick Blute and Peter Selinger, editors, Electronic Notes in Theoretical Computer Science, volume 69. Elsevier, 2003.

[Isi96] A. Isidori. Nonlinear Control Systems. Springer-Verlag, third edition, 1996

[JNW96] A. Joyal, M. Nielsen, and G. Winskel. Bisimulation from open maps. Information and Computation, 127:164-185, 1996.

[Jur97] Velimir Jurdjevic. Geometric Control Theory. Number 51 in Cambridge Studies in Advanced Mathematics. Cambridge University Press, 1997.

[Lan71] Saunders Mac Lane. Categories for the Working Mathematician. Springer-Verlag, 1971.

[Lew00] Andrew D. Lewis. The category of affine connection control systems. In Proceedings of the 39th IEEE conference on decision and control, pages 1260-1265, Sydney, Australia, December 2000.

[NvdS95] H. Nijmeijer and A.J. van der Schaft. Nonlinear Dynamical Control Systems. Springer-Verlag, 1995.

[Pap03] George J. Pappas. Bisimilar linear systems. Automatica, 39(12):2035-2047, December 2003.

[PLS00] G. J. Pappas, G. Lafferriere, and S. Sastry. Hierarchically consistent control systems. IEEE Transactions on Automatic Control, 45(6):1144-1160, June 2000.

[SJK97] R. Sepulchre, M. Jankovic, and P. V. Kokotovic. Constructive Nonlinear Control. Communications and Control Engineering. Springer-Verlag, New York, January 1997.

[Tab05] Paulo Tabuada. Local factorization of trajectory lifting morphisms for single-input affine control systems. 2005. Submitted for publication. Available at http: / / www. nd. edu/ ptabuada.

[TP04] P. Tabuada and G. J. Pappas. Bisimilar control affine systems. Systems and Control Letters, 52(1):49-58, 2004.

[TP05a] P. Tabuada and G. Pappas. Hierarchical trajectory generation for a class of nonlinear systems. Automatica, 41(4):701-708, 2005.

[TP05b] P. Tabuada and G. J. Pappas. Quotients of fully nonlinear control systems. SIAM Journal on Control and Optimization, 43(5):1844-1866, 2005.

[vdS04] A. van der Schaft. Equivalence of dynamical systems by bisimulation. IEEE Transactions on Automatic Control, 49(12):21602172, 2004. 